\title{
AUTOMATIC OPTIMIZATION TECHNIQUES APPLIED TO A LARGE RANGE OF INDUSTRIAL TEST CASES
}

\author{
R.Ducloux $^{1}$, L. Fourment ${ }^{2}$, S. Marie $^{1}$, D. Monnereau ${ }^{3}$ \\ ${ }^{1}$ Transvalor S. A., Sophia-Antipolis, France \\ ${ }^{2}$ Mines ParisTech - CEMEF (Centre for Material Forming), Sophia Antipolis, France \\ ${ }^{3}$ Bollhoff-Ottalu, France
}

\begin{abstract}
The use of material processing numerical simulation has spread widely in recent years in the engineering industry. It allows a strategy of trial and error to improve virtual processes without incurring material costs or interrupting production and therefore save a lot of money. On the other hand, it requires user time to analyze the results, adjust the operating conditions and restart the simulation. Automatic optimization seems the perfect complement to simulation. Evolutionary Algorithm coupled with metamodelling makes it possible to obtain industrially relevant results on a very large range of applications within a few tens of simulations and without any specific automatic optimization technique knowledge. In the frame of the LOGIC ANR French project, ten industrial partners have been selected to cover the different area of the mechanical forging industry and provide different examples of the forming simulation tools. An optimization module, fully embedded within the Forge2009 IHM, makes possible to cover all the defined examples, and the use of new multicore hardware to compute several simulations at the same time reduces the needed time dramatically. The presented examples demonstrate the method versatility. They include billet shape optimization of a common rail and the cogging of a bar.
\end{abstract}

KEYWORDS: Optimization, Metamodelling, Evolution Strategy, FORGE software, Forging, Open die forging, Closed-die forging

\section{INTRODUCTION}

The use of material processing numerical simulation has spread widely in recent years in the engineering industry. It allows a strategy of trial and error to improve virtual processes without incurring material costs or interrupting production and therefore save a lot of money. On the other hand, it requires user time to analyze the results, adjust the operating conditions and restart the simulation. Therefore this process is often interrupted before the optimum is reached for lack of time. Automatic optimization seems the perfect complement to simulation. Despite this, if nice academic example can be found, there is very little coupled use in the industry. The reasons are many:

(a) Difficulty in applying optimization techniques to a complex reality and to formalize a particular optimality criterion which is often a mix between the quality of the part (criteria based on thermo-mechanical history and lack of default) and its cost (the amount of material used, deterioration of tools, equipment selection).

(b) Complexity of triple coupling between the core optimization, simulation software and possibly CAD software. (c) Difficulties in the form of objective functions that make difficult the use of first-order algorithms and the time of evaluation which could exclude the zero-order algorithms.

The French founded ANR Logic project gathers ten FORGE end users, Cemef and Transvalor. It aims to demonstrate that, using Metamodelling based Evolution Strategies, it is possible to obtain industrially relevant results on a very large range of applications within a few tens of simulations and without any specific automatic optimization technique knowledge. To achieve this proof, the ten industrial partners have been selected to cover the different area of the mechanical forging industry and each of them came with 2 or 3 different examples typical of its own use of the forming simulation tools. Two years after the beginning of the project, the first goal is reached. The optimization module, fully embedded within the Forge2009 IHM makes possible to cover all the defined example and the use of new multicore hardware to compute several simulations at the same time reduces the needed time dramatically.

The examples presented here have been selected among the project test case to demonstrate the versatility of the

\footnotetext{
* Corresponding author: Dr Richard Ducloux, Technical Manager, TRANSVALOR, Parc de haute Technologie, Sophia Antipolis, 694 av du Dr Maurice Donat, 06255 Mougins Cedex FRANCE, Phone: 33 (0)4 92924215 - Fax: 33 (0)4 92924201 , richard.ducloux@transvalor.com
} 
method. They include billet shape optimization of a common rail and the cogging of a bar.

\section{MAES algorithm}

Meta-model Assisted Evolution Strategies (MAES) are regarded as quite robust algorithms with respect to local extrema. They make it possible to solve the most complex optimisation problems. Evolutionary algorithms (ES) typically consist of three operators: selection, recombination and mutation. Their cost is usually quite high in terms of function evaluations. MAES proposed by Emmerich et al [1] combines an ES with Kriging meta-models to reduce the number of function evaluations. An overview of MAES is depicted in Figure 1. It starts by randomly choosing an initial population of (a minimum of) 2 times the number of optimization parameters. The number of parents, $\lambda$, is set to (a minimum of) 2 times the number of optimization parameters, while the number of children, $\mu$, is set to (a minimum of) 4 times $\lambda$.

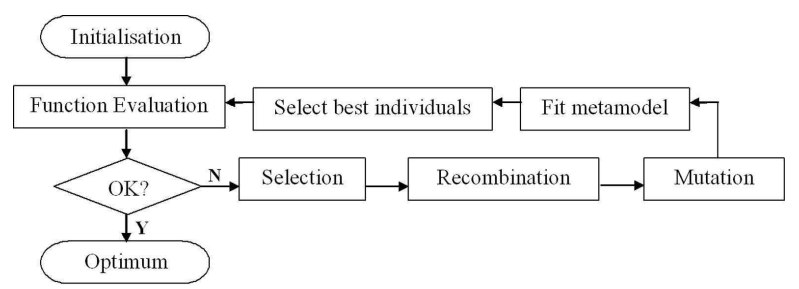

Figure 1: Overview of MAES.

After having run the F.E. simulations for the initial population, the $\lambda$ best settings are selected, recombined and mutated to yield $\mu$ children. The results of the previously performed F.E. calculations are used to fit a Kriging meta-model, so instead of running the expensive F.E. calculations for the $\mu$ children, the results are first estimated. The objective function values $f$ are not directly approximated by $\tilde{f}$, but by $\tilde{f}-\Delta \tilde{f}$, where $\Delta \tilde{f}$ is the Root Mean Square Error of the Kriging approximation. $\tilde{f}-\Delta \tilde{f}$ is the merit function. Based on these predictions, only the $20 \%$ best individuals are actually evaluated by running the F.E. simulations. In this way, the meta-modelling technique saves $80 \%$ of time-consuming calculations. The Kriging meta-model is then updated, and this procedure is repeated until the maximum number of F.E. simulations is reached.

According to the problem size, each F.E. calculation can be run on a certain number of processors, $N_{F . E \text {. It is }}$ useless to appeal to too many processors because the parallel efficiency decreases after a certain number. On the other hand, it is quite efficient to benefit from the parallel structure of the ES, by running the $\lambda$ (or a portion of $\lambda$ )F.E. simulations at the same time on different machines. Consequently, $\lambda N_{\text {F.E. }}$ processors can be used for the parallel calculations, with a very high efficiency.

\section{FORGING OF A COMMON RAIL}

The first application regards the shape optimization of a cylindrical preform (see Figure 2) that is used to produce a common rail by two steps of closed die forging (see Figure 3). The axisymmetric preform is parameterized using 3 parameters: its larger and smaller diameters, and its length. The ratios between the different parts of the shape are kept constant. The optimization objective is to minimize the mass of the component (i.e. of the preform) under the constraint of forming the right component (i.e. of perfectly filling the active part of forging dies - i.e. without taking into account the flash). The constraint is handled by a penalty approach. The objective and constraint function are aggregated into a single objective function.

The MAES algorithm was set-up with a population of 45 individuals (9 parents and 36 children). At each generation, only 9 exact calculations $(20 \%)$ are carried out on the available cluster of 32 cores - consisting of 8 nodes of 4-core processors. For each individual, the two forging steps are calculated in parallel on the 4 cores of each node, while the 9 individuals are calculated in parallel on a maximum of 5 nodes, in two batches. In a first approach, the maximum number of generations was set to 6 , for a total of 54 exact forging simulations.

Figure 2 shows two preform shapes that have been tested by the algorithm. They have similar mass but very different aspect ratios. In Figure 4, four simulation results are presented. The bottom left case represents an unfilled configuration, where the component details do not appear. The bottom right case allows proper filling, but the mass is quite large. In spite of its mass that is heavier than the top right solution, the top left solution does not provide a proper filling of the thin arm. The evolution of both the constraint and objective functions along with the genetic generations are plotted in Figure 5 , while Figure 6 more clearly shows the weight reduction. They show the convergence of the MAES algorithm toward a quite satisfactory solution within only 6 generations; the filling constraint is satisfied after the first generation of the algorithm, and the following generations allows minimizing the preform mass, from $26.1 \mathrm{~kg}$ to $25.7 \mathrm{~kg}$.

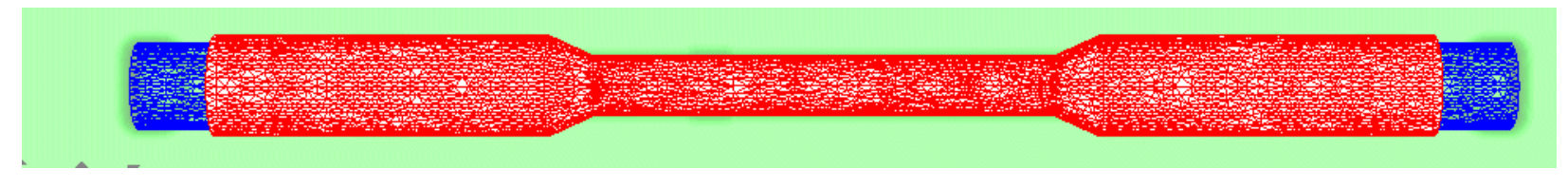

Figure 2: Two preform shapes utilized for the forging of the common rail component. 


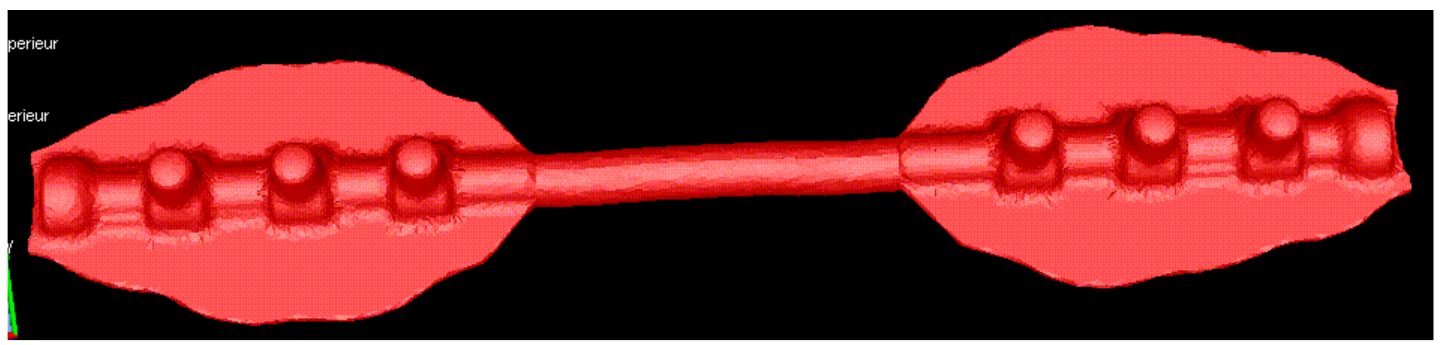

Figure 3: Closed-die forging of a "common rail" component - end of the second step of forging.
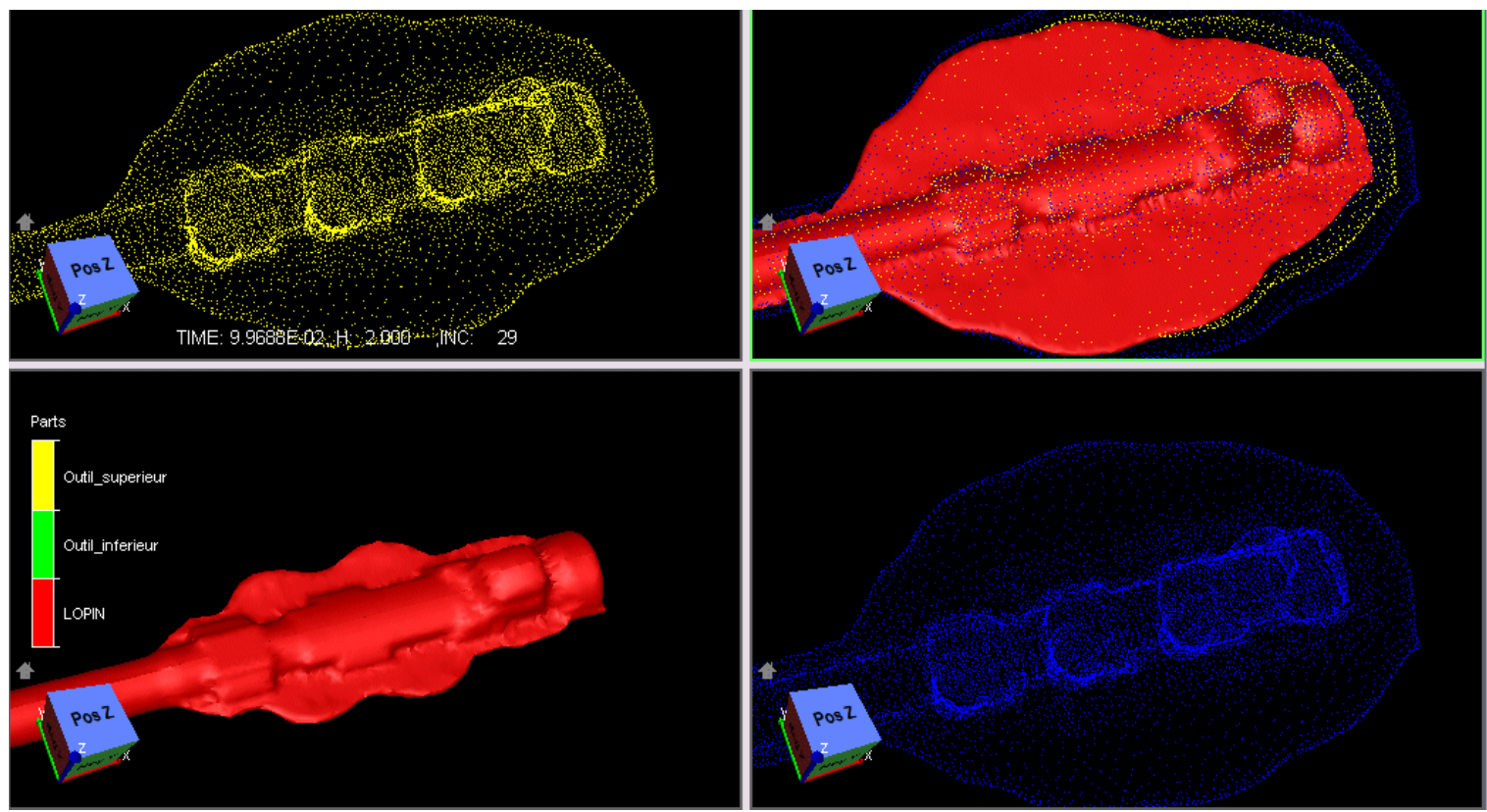

Figure 4: Final shape of the component for different results of optimization.

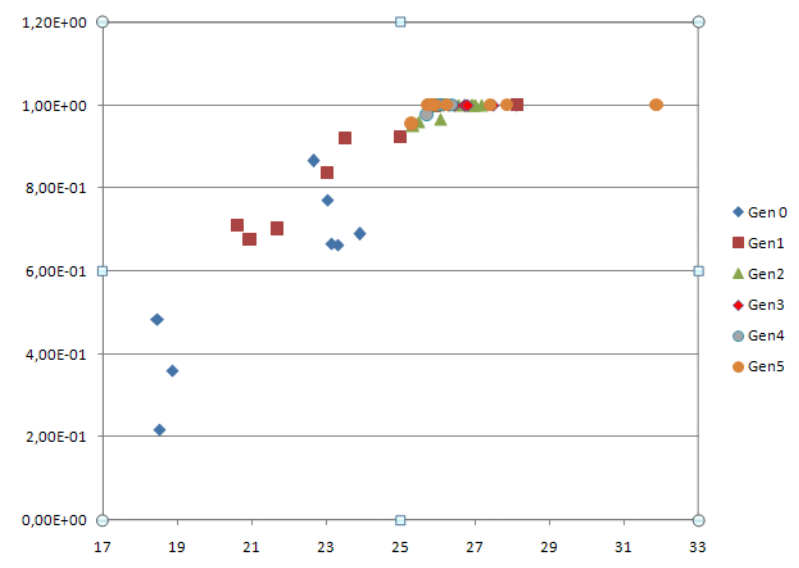

Figure 5: Pareto plot in the mass (horizontal axis) / die filling (vertical axis) frame of the calculated solutions at the different generations of MAES.

\section{COGGING}

After cooling, a cast billet exhibits localized porosities that can be calculated using a Yamanaka model (see Figure 7). In order to close these porosities, the billet is open-die forged in order to get a square section by 12 steps of cogging with a $90^{\circ}$ rotation between each.

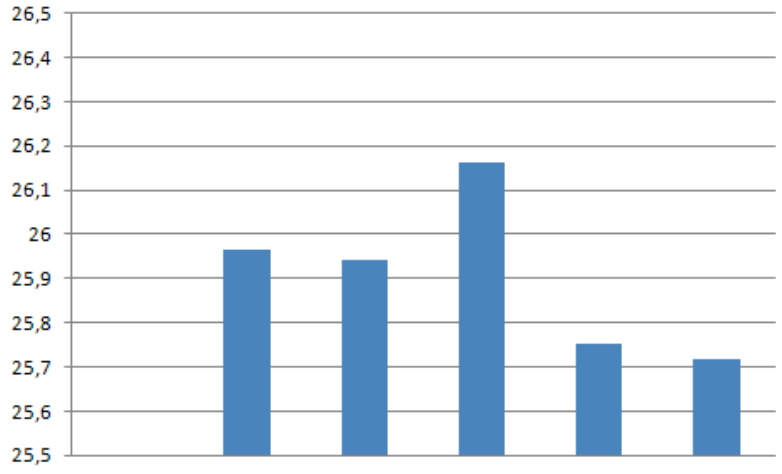

Figure 6: Minimum weight of the individual of each generation satisfying the filling constraint.

Figure 8 shows the reduction of these porosities at different steps of cogging. The optimization problem consists in determining the final heights of the successive strokes $n^{\circ} 1,3,5,7,9$ (the height of stroke $\mathrm{n}^{\circ} 2$ is equal to the one of stroke $\mathrm{n}^{\circ} 1$ and so on), which provide 5 optimization parameters, in order to get a prescribed section after the $12^{\text {th }}$ stroke (the remaining two strokes have prescribed heights) and to optimize the material deformation for closing the porosities. 


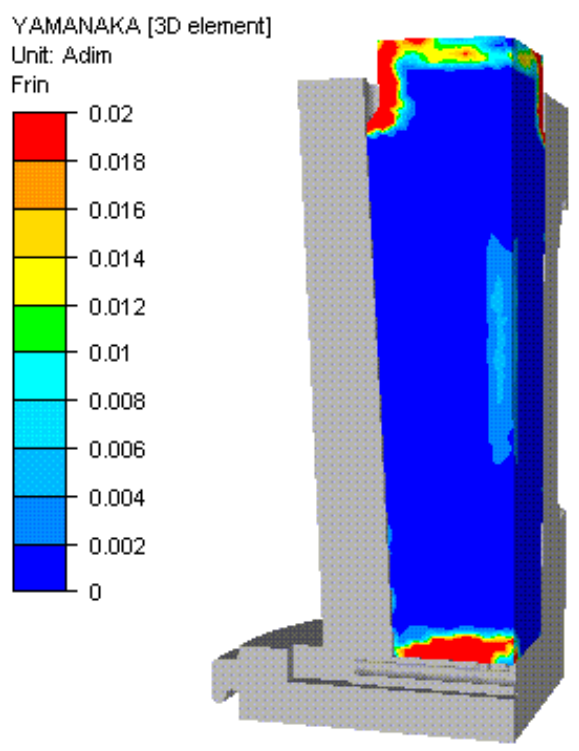

Figure 7: Isovalues of the Yamanaka porosity model at the end of cooling of the initial billet.
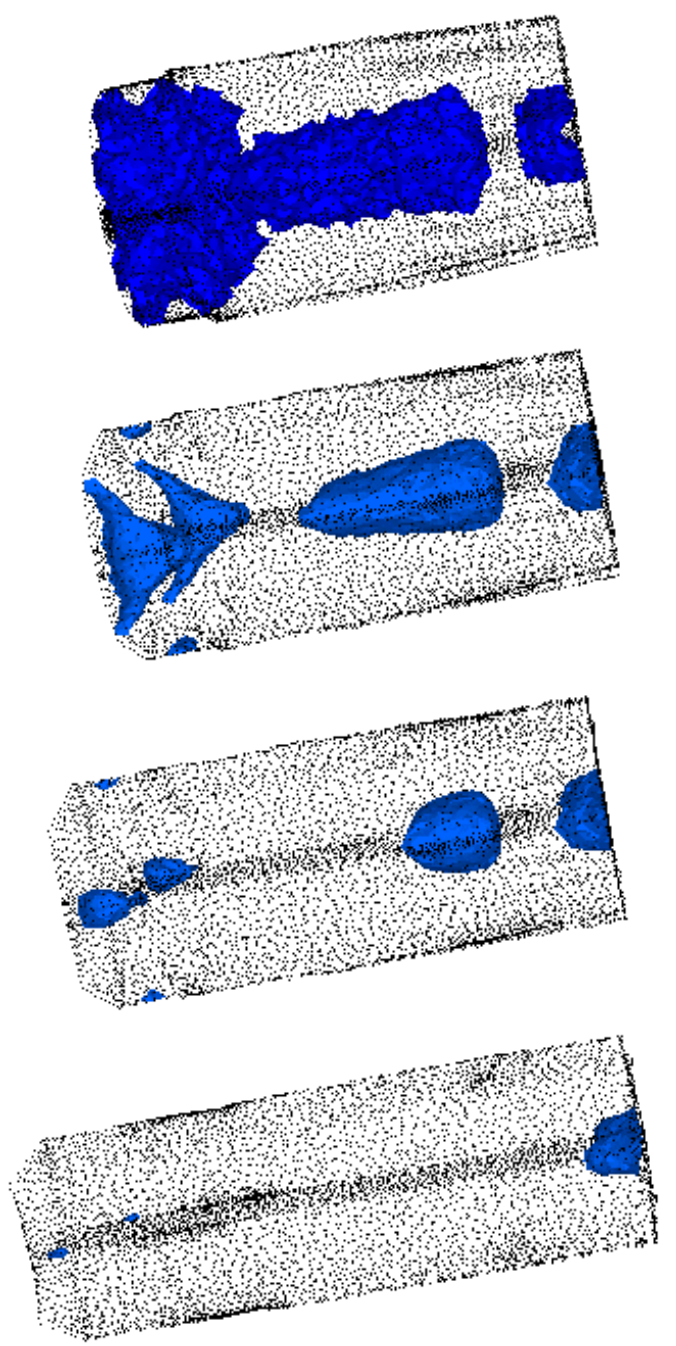

Figure 8: Reduction of the 0.2 isovalue of porosity at different phases of the cogging strokes.
The MAES algorithm was set-up in order that 15 exact calculations were carried out in parallel (in two batches) at each generation, each series of 12 forging steps being calculated in parallel on the 4 cores processors. The maximum number of generations was set to 5 , for a total of 75 exact forging simulations.

The results of this first batch of calculations were quite satisfactory, as can be seen in Figure 9 that shows the optimal obtained solution, in comparison to a less satisfactory solution. The material porosities have been almost totally closed, except at the two ends of the bar, where they were initially larger and where the process efficiency is lower.
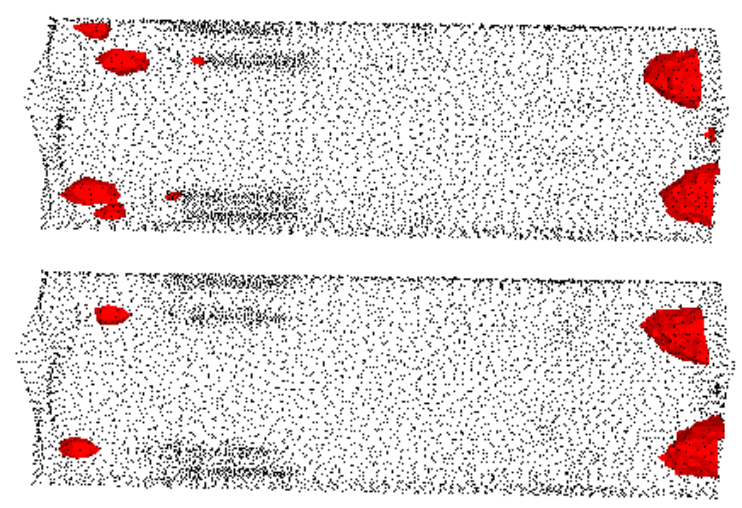

Figure 9: 0.2 isovalue of porosity at the end of cogging for a good (top) and for the optimal (bottom) solutions.

\section{CONCLUSION}

Optimization algorithms based on meta-modelling techniques can be applied to actual and very complex metal forming problems. The computational time is well handled by the parallelization of the evolutionary algorithm, in addition to the parallelization of the F.E. software itself. Complex design problems can be tackled, like the presented preform shape design for the forging of a common rail and the achievement of an attractive mass reduction, or like the process design for the cogging of a bar with a large number of parameters and the obtaining of a satisfactory micro-structure.

\section{ACKNOWLEDGEMENT}

This work has been carried out in the frame of the LOGIC ANR program, which support is gratefully acknowledged.

\section{REFERENCE}

[1] M. Emmerich, A. Giotis, M. Özdemir, T. Bäck, K. Giannakoglou. Metamodel-assisted evolution strategies, Int. Conf. on parallel problem solving from nature Springer, Berlin, GERMANY, 2002 